\title{
On the symmetric positive solutions of nonlinear fourth or- der ordinary differential equations with four-point bound- ary value conditions: a fixed point theory approach
}

\author{
Md. Asaduzzaman ${ }^{a, *}$, Md. Zulfikar Ali ${ }^{b}$ \\ ${ }^{a}$ Department of Mathematics, Islamic University, Kushtia-7003, Bangladesh. \\ ${ }^{b}$ Department of Mathematics, University of Rajshahi, Rajshahi-6205, Bangladesh.
}

\begin{abstract}
The purpose of this paper is to investigate the existence of symmetric positive solutions of the following nonlinear fourth order system of ordinary differential equations

$$
\left\{\begin{array}{l}
-\mathfrak{u}^{(4)}(t)=f(t, v) \\
-v^{(4)}(t)=g(t, u), \quad t \in[0,1]
\end{array}\right.
$$

with the four-point boundary value conditions

$$
\left\{\begin{array}{l}
u(t)=u(1-t), \mathfrak{u}^{\prime \prime \prime}(0)-\mathfrak{u}^{\prime \prime \prime}(1)=\mathfrak{u}^{\prime \prime}\left(\mathrm{t}_{1}\right)+\mathfrak{u}^{\prime \prime}\left(\mathrm{t}_{2}\right) \\
v(\mathrm{t})=v(1-\mathrm{t}), v^{\prime \prime \prime}(0)-v^{\prime \prime \prime}(1)=v^{\prime \prime}\left(\mathrm{t}_{1}\right)+v^{\prime \prime}\left(\mathrm{t}_{2}\right), 0<\mathrm{t}_{1}<\mathrm{t}_{2}<1
\end{array}\right.
$$

By applying Krasnoselskii's fixed point theorem and under suitable conditions, we establish the existence of at least one or at least two symmetric positive solutions of the above mentioned fourth order four-point boundary value problem in cone. Some particular examples are provided to support the analytic proof.
\end{abstract}

Keywords: Fourth order four-point boundary value problem, existence of symmetric positive solution, Krasnoselskii's fixed point theorem, Green's function.

2010 MSC: 34B10, 34B15, 34B18.

(C)2020 All rights reserved.

\section{Introduction}

Boundary value problems for fourth order ordinary differential equations are used to describe a huge number of physical, biological and chemical phenomena, see for instance [1, 7, 11, 15, 19, 21, 29] and references therein. In recent years, there has been a noticeable interest on the solution-existence prediction methods and it is well established that the fixed point techniques the are most important methods for

\footnotetext{
*Corresponding author

Email addresses: masad_iu_math@yahoo.com (Md. Asaduzzaman), alimath1964@gmail.com (Md. Zulfikar Ali)

doi: $10.22436 /$ jnsa.013.06.06
}

Received: 2018-10-20 Revised: 2020-02-07 Accepted: 2020-03-03 
predicting the existence of solutions of ordinary differential equations with boundary conditions, see for instance $[2-4,6,16,23,24]$ and their cited references. In the last few decades, symmetric positive solution of two-point, three-point and four-point boundary value problems for second order, third order, fourth order as well as higher order has extensively been studied by using various techniques, see for instance $[4,5,8,9,12-14,17,18,20,22,25-28]$ and references therein, but only a small number of works have been established on the existence of symmetric positive solution of system of boundary value problem (BVP for short).

Li and Zhang [13], and Henderson and Thompson [12] studied the multiple symmetric positive solutions of second order system of ordinary differential equations. Feng et al. [8] studied the existence of multiple symmetric positive solutions to the system of four-point boundary-value problems with onedimensional P-Laplacian. Cetin and Topal [5], studied the symmetric positive solutions of fourth order boundary value problems for an increasing homeomorphism and homomorphism on time-scales. In $[17,18]$, Haidong studied symmetric positive solutions of second order system of nonlinear ordinary differential equations with three-point and four-point boundary conditions by using Krasnoselskii's fixed point theorem [10] in cone. Recently, Feng et al. [9] established the symmetric positive solutions for fourth-order n-dimensional differential equations with $\mathrm{m}$-Laplace systems. So, from the above discussion it is clear that there is a certain gap in literature about the solution procedure on the existence of symmetric positive solutions of system of nonlinear fourth order ordinary differential equations (SNLFOODEs for short) with four-point boundary conditions by applying fixed point theorem. To fill up this certain gap, here we have studied the existence of symmetric positive solutions of following SNLFOODEs

$$
\left\{\begin{array}{l}
-u^{(4)}(t)=f(t, v), \\
-v^{(4)}(t)=g(t, u), \quad t \in[0,1]
\end{array}\right.
$$

with the four-point boundary value conditions

$$
\left\{\begin{array}{l}
u(t)=u(1-t), u^{\prime \prime \prime}(0)-u^{\prime \prime \prime}(1)=u^{\prime \prime}\left(t_{1}\right)+u^{\prime \prime}\left(t_{2}\right), \\
v(t)=v(1-t), v^{\prime \prime \prime}(0)-v^{\prime \prime \prime}(1)=v^{\prime \prime}\left(t_{1}\right)+v^{\prime \prime}\left(t_{2}\right), 0<t_{1}<t_{2}<1,
\end{array}\right.
$$

where, both $f:[0,1] \times \mathbf{R}^{+} \rightarrow \mathbf{R}^{+}, g:[0,1] \times \mathbf{R}^{+} \rightarrow \mathbf{R}^{+}$are continuous, both $f(t, v)$ and $g(t, u)$ are symmetric on $[0,1]$ and $f(t, 0) \equiv 0, g(t, 0) \equiv 0$, by applying Krasnoselskii's fixed point theorem under some predefined hypothesis in cone. The rest of this paper is organized as follows. In Section 2, we provide some necessary definitions, lemmas and theorems associated with BVP given by (1.1) and (1.2). In Section 3, the main results will be stated and proved. Finally, we give some examples to illustrate our main results.

\section{Preliminary}

In this section, we recall some essential definitions and establish some lemmas which help us to establish our main results. Throughout this paper $C[0,1]$ denotes the set of continuous functions on $[0,1]$.

Definition 2.1 $([18,26])$. Let $(B,\|\|$.$) be a real Banach space and K$ be a nonempty closed convex subset of

$B$. This subset $B K$ is called a cone of $B$ if it satisfies the following conditions:

(i) $x \in K, \mu>0$ implies $\mu x \in K$;

(ii) $x \in K,-x \in K$ implies $x=0$.

Example 2.2. A funnel is an example of a cone.

Definition 2.3 ([18]). The function $u$ is said to be concave on $[0,1]$ if

$$
u\left(\lambda t_{1}+(1-\lambda) t_{2}\right) \geqslant \lambda u\left(t_{1}\right)+(1-\lambda) u\left(t_{2}\right),
$$

for all $\lambda, t_{1}, t_{2} \in[0,1]$. 
Definition 2.4 ([18]). The function $u$ is said to be symmetric on $[0,1]$, if $u(t)=u(1-t)$, for all $t \in[0,1]$.

Lemma 2.5. Assume $\mathrm{t}_{1}$ and $\mathrm{t}_{2}$ are two nonnegative constants satisfying $0 \leqslant \mathrm{t}_{1}<\mathrm{t}_{2} \leqslant 1$. If $\mathrm{h}(\mathrm{t}) \in \mathrm{C}[0,1]$ is symmetric on $[0,1]$, then the following second order four-point BVP

$$
\left\{\begin{array}{l}
u^{\prime \prime}=-h(t), \quad 0 \leqslant t \leqslant 1, \\
u(t)=u(1-t), u^{\prime}(0)-u^{\prime}(1)=u\left(t_{1}\right)+u\left(t_{2}\right),
\end{array}\right.
$$

has a unique symmetric solution

$$
u(t)=\int_{0}^{1} G(t, s) h(s) d s
$$

where $\mathrm{G}(\mathrm{t}, \mathrm{s})$ is the Green function of

$$
\begin{gathered}
\qquad\left\{\begin{array}{l}
u^{\prime \prime}=0,0 \leqslant t \leqslant 1, \\
u(t)=u(1-t), u^{\prime}(0)-u^{\prime}(1)=u\left(t_{1}\right)+u\left(t_{2}\right),
\end{array}\right. \\
\text { i.e., } G(t, s)=G_{1}(t, s)+G_{2}(s) \text { and } G_{1}(t, s)= \begin{cases}t(1-s), & 0 \leqslant t<s \leqslant 1, \\
s(1-t), & 0 \leqslant s \leqslant t \leqslant 1,\end{cases} \\
G_{2}(s)=\left\{\begin{array}{l}
\frac{1}{2}\left[\left(t_{1}-s\right)+\left(t_{2}-s\right)-t_{1}(1-s)-t_{2}(1-s)+1\right], \quad 0 \leqslant s \leqslant t_{1}, \\
\frac{1}{2}\left[\left(t_{2}-s\right)-t_{1}(1-s)-t_{2}(1-s)+1\right], \quad t_{1} \leqslant s \leqslant t_{2}, \\
\frac{1}{2}\left[-t_{1}(1-s)-t_{2}(1-s)+1\right], \quad t_{2} \leqslant s \leqslant 1 .
\end{array}\right.
\end{gathered}
$$

Proof. From (2.1), we have $\mathfrak{u}^{\prime \prime}=-\mathrm{h}(\mathrm{t})$. Integrating from 0 to 1 , we get

$$
u^{\prime}(t)=-\int_{0}^{1} h(s) d s+a_{1} .
$$

Since $u(t)=u(1-t) \Rightarrow u^{\prime}(t)=-u^{\prime}(1-t)$, then we obtain $-\int_{0}^{t} h(s) d s+a_{1}=\int_{0}^{1-t} h(s) d s-a_{1}$, which gives

$$
\begin{aligned}
a_{1}=\frac{1}{2}\left[\int_{0}^{t} h(s) d s+\int_{0}^{1-t} h(s) d s\right] & =\frac{1}{2}\left[\int_{0}^{t} h(s) d s-\int_{1}^{t} h(1-s) d s\right] \\
& =\frac{1}{2}\left[\int_{0}^{t} h(s) d s+\int_{t}^{1} h(s) d s\right]=\int_{0}^{1}(1-s) h(s) d s .
\end{aligned}
$$

Putting the value of $a_{1}$ in (2.2), we get

$$
u^{\prime}(t)=-\int_{0}^{t} h(s) d s+\int_{0}^{1}(1-s) h(s) d s .
$$

Now integrating both sides of (2.2), we obtain

$$
u(t)=-\int_{0}^{t}(t-s) h(s) d s+t \int_{0}^{1}(1-s) h(s) d s+a_{2} .
$$

Using (2.3) and (2.4), in the boundary value condition of (2.1), we yield

$$
\begin{aligned}
\int_{0}^{1} h(s) d s= & -\int_{0}^{t_{1}}\left(t_{1}-s\right) h(s) d s+t_{1} \int_{0}^{1}(1-s) h(s) d s+2 a_{2} \\
& -\int_{0}^{t_{2}}\left(t_{2}-s\right) h(s) d s+t_{2} \int_{0}^{1}(1-s) h(s) d s,
\end{aligned}
$$


which gives

$$
\begin{aligned}
a_{2}= & \frac{1}{2} \int_{0}^{1} h(s) d s+\frac{1}{2} \int_{0}^{t_{1}}\left(t_{1}-s\right) h(s) d s-\frac{1}{2} t_{1} \int_{0}^{1}(1-s) h(s) d s \\
& +\frac{1}{2} \int_{0}^{t_{2}}\left(t_{2}-s\right) h(s) d s-\frac{1}{2} t_{2} \int_{0}^{1}(1-s) h(s) d s \\
= & \frac{1}{2} \int_{0}^{t_{1}}\left[\left(t_{1}-s\right)+\left(t_{2}-s\right)-t_{1}(1-s)-t_{2}(1-s)+1\right] h(s) d s \\
& +\frac{1}{2} \int_{t_{1}}^{t_{2}}\left[\left(t_{2}-s\right)-t_{1}(1-s)-t_{2}(1-s)+1\right] h(s) d s \\
& +\frac{1}{2} \int_{t_{2}}^{1}\left[-t_{1}(1-s)-t_{2}(1-s)+1\right] h(s) d s .
\end{aligned}
$$

Putting the value of $a_{2}$ in (2.4), we get

$$
\begin{aligned}
u(t)= & -\int_{0}^{t}(t-s) h(s) d s+t \int_{0}^{1}(1-s) h(s) d s \\
& +\frac{1}{2} \int_{0}^{t_{1}}\left[\left(t_{1}-s\right)+\left(t_{2}-s\right)-t_{1}(1-s)-t_{2}(1-s)+1\right] h(s) d s \\
& +\frac{1}{2} \int_{t_{1}}^{t_{2}}\left[\left(t_{2}-s\right)-t_{1}(1-s)-t_{2}(1-s)+1\right] h(s) d s \\
& +\frac{1}{2} \int_{t_{2}}^{1}\left[-t_{1}(1-s)-t_{2}(1-s)+1\right] h(s) d s \\
= & \int_{0}^{1} G_{1}(t, s) h(s) d s+\int_{0}^{1} G_{2}(s) h(s) d s=\int_{0}^{1} G(t, s) h(s) d s,
\end{aligned}
$$

which is the unique symmetric solution of the BVP given by (2.1). This completes the proof.

Lemma 2.6. Assume $\mathrm{t}_{1}$ and $\mathrm{t}_{2}$ are two nonnegative constants satisfying $0 \leqslant \mathrm{t}_{1}<\mathrm{t}_{2} \leqslant 1$. If $\mathrm{h}^{*}(\mathrm{t}) \in \mathrm{C}[0,1]$ is symmetric on $[0,1]$, then the following fourth order four-point BVP

$$
\left\{\begin{array}{l}
u^{(4)}=-h^{*}(t), \quad 0 \leqslant t \leqslant 1 \\
u(t)=u(1-t), \quad u^{\prime \prime \prime}(0)-u^{\prime \prime \prime}(1)=u^{\prime \prime}\left(t_{1}\right)+u^{\prime \prime}\left(t_{2}\right),
\end{array}\right.
$$

has a unique symmetric solution

$$
u(t)=\int_{0}^{1} G(t, s)\left(\int_{0}^{1} G^{*}(s, r) h^{*}(r) d r\right) d s
$$

where $\mathrm{G}(\mathrm{t}, \mathrm{s})=\mathrm{G}_{1}(\mathrm{t}, \mathrm{s})+\mathrm{G}_{2}(\mathrm{~s}), \mathrm{G}_{1}(\mathrm{t}, \mathrm{s})= \begin{cases}\mathrm{t}(1-\mathrm{s}), & 0 \leqslant \mathrm{t}<\mathrm{s} \leqslant 1 \\ \mathrm{~s}(1-\mathrm{t}), & 0 \leqslant \mathrm{~s} \leqslant \mathrm{t} \leqslant 1,\end{cases}$

$$
G_{2}(s)= \begin{cases}\frac{1}{2}\left[\left(t_{1}-s\right)+\left(t_{2}-s\right)-t_{1}(1-s)-t_{2}(1-s)+1\right], & 0 \leqslant s \leqslant t_{1}, \\ \frac{1}{2}\left[\left(t_{2}-s\right)-t_{1}(1-s)-t_{2}(1-s)+1\right], & t_{1} \leqslant s \leqslant t_{2}, \\ \frac{1}{2}\left[-t_{1}(1-s)-t_{2}(1-s)+1\right], & t_{2} \leqslant s \leqslant 1\end{cases}
$$

and $\mathrm{G}^{*}(s, r)=\mathrm{G}_{1}^{*}(s, r)+\mathrm{G}_{2}^{*}(r), \mathrm{G}_{1}^{*}(s, r)= \begin{cases}s(1-r), & 0 \leqslant s<r \leqslant 1, \\ r(1-s), & 0 \leqslant r \leqslant s \leqslant 1,\end{cases}$ 


$$
G_{2}^{*}(r)= \begin{cases}\frac{1}{2}\left[\left(t_{1}-r\right)+\left(t_{2}-r\right)-t_{1}(1-r)-t_{2}(1-r)+1\right], & 0 \leqslant r \leqslant t_{1}, \\ \frac{1}{2}\left[\left(t_{2}-r\right)-t_{1}(1-r)-t_{2}(1-r)+1\right], & t_{1} \leqslant r \leqslant t_{2}, \\ \frac{1}{2}\left[-t_{1}(1-r)-t_{2}(1-r)+1\right], & t_{2} \leqslant r \leqslant 1 .\end{cases}
$$

Proof. To prove this lemma first, let $\mathrm{u}^{\prime \prime}=w(t)$ then we have

$$
u(t)=\int_{0}^{1} G(t, s) w(s) d s,
$$

and the BVP given by (2.5) is equivalent to the following BVP

$$
\begin{cases}w^{\prime \prime}=-h^{*}(t), & 0 \leqslant t \leqslant 1 \\ w(t)=w(1-t), & w^{\prime}(0)-w^{\prime}(1)=w\left(t_{1}\right)+w\left(t_{2}\right) .\end{cases}
$$

Now, by Lemma 2.5, we know that the BVP given by (2.7) is equivalent to the following integral equation

$$
w(t)=\int_{0}^{1} G^{*}(t, r) h^{*}(r) d r
$$

where $G^{*}(t, r)$ is the Green function of

$$
\begin{aligned}
&\left\{\begin{array}{l}
w^{\prime \prime}=0,0 \leqslant t \leqslant 1, \\
w(t)=w(1-t), w^{\prime}(0)-w^{\prime}(1)=w\left(t_{1}\right)+w\left(t_{2}\right),
\end{array}\right. \\
& \text { i.e., } G^{*}(t, r)=G_{1}^{*}(t, r)+G_{2}^{*}(r) \text { and } G_{1}^{*}(t, r)= \begin{cases}t(1-r), & 0 \leqslant t<r \leqslant 1, \\
r(1-t), & 0 \leqslant r \leqslant t \leqslant 1,\end{cases} \\
& G_{2}^{*}(s)= \begin{cases}\frac{1}{2}\left[\left(t_{1}-r\right)+\left(t_{2}-r\right)-t_{1}(1-r)-t_{2}(1-r)+1\right], & 0 \leqslant r \leqslant t_{1}, \\
\frac{1}{2}\left[\left(t_{2}-r\right)-t_{1}(1-r)-t_{2}(1-r)+1\right], & t_{1} \leqslant r \leqslant t_{2}, \\
\frac{1}{2}\left[-t_{1}(1-r)-t_{2}(1-r)+1\right], & t_{2} \leqslant r \leqslant 1 .\end{cases}
\end{aligned}
$$

Finally, from the combination of (2.6) and (2.8), we obtain

$$
u(t)=\int_{0}^{1} G(t, s)\left(\int_{0}^{1} G^{*}(s, r) h^{*}(r) d r\right) d s .
$$

This completes the lemma.

Lemma 2.7. Let $\mathrm{m}_{\mathrm{G}_{2}}=\min \left\{\mathrm{G}_{2}\left(\mathrm{t}_{1}\right), \mathrm{G}_{2}\left(\mathrm{t}_{2}\right)\right\}, \mathrm{l}=\frac{4 \mathrm{~m}_{\mathrm{G}_{2}}}{4 \mathrm{~m}_{\mathrm{G}_{2}}+1}$, then for all $\mathrm{t}, \mathrm{s} \in[0,1]$, the Green function $\mathrm{G}(\mathrm{t}, \mathrm{s})$ satisfies the inequality

$$
\mathrm{lG}(\mathrm{s}, \mathrm{s}) \leqslant \mathrm{G}(\mathrm{t}, \mathrm{s}) \leqslant \mathrm{G}(\mathrm{s}, \mathrm{s}),
$$

where $\mathrm{G}(\mathrm{t}, \mathrm{s}), \mathrm{G}(\mathrm{s}, \mathrm{s})$ and $\mathrm{G}_{2}(\mathrm{~s})$ are defined as like the Lemma 2.6 .

Proof. Since, $t, s \in[0,1]$, then we have

$$
\begin{aligned}
\mathrm{G}(\mathrm{t}, \mathrm{s})=\mathrm{G}_{1}(\mathrm{t}, \mathrm{s})+\mathrm{G}_{2}(\mathrm{~s}) \geqslant \mathrm{G}_{2}(\mathrm{~s}) & =\frac{1}{4 \mathrm{~m}_{\mathrm{G}_{2}}+1} \mathrm{G}_{2}(\mathrm{~s})+\frac{4 \mathrm{~m}_{\mathrm{G}_{2}}}{4 \mathrm{~m}_{\mathrm{G}_{2}}+1} \mathrm{G}_{2}(\mathrm{~s}) \\
& \geqslant \frac{1}{4} \cdot \frac{4 m_{\mathrm{G}_{2}}}{4 \mathrm{~m}_{\mathrm{G}_{2}}+1}+\frac{4 \mathrm{~m}_{\mathrm{G}_{2}}}{4 \mathrm{~m}_{\mathrm{G}_{2}}+1} \mathrm{G}_{2}(\mathrm{~s}) \\
& \geqslant s(1-\mathrm{s}) \cdot \frac{4 \mathrm{~m}_{\mathrm{G}_{2}}}{4 \mathrm{~m}_{\mathrm{G}_{2}}+1}+\frac{4 \mathrm{~m}_{\mathrm{G}_{2}}}{4 \mathrm{~m}_{\mathrm{G}_{2}}+1} \mathrm{G}_{2}(\mathrm{~s}) \\
& =l \mathrm{G}_{1}(\mathrm{~s}, \mathrm{~s})+l \mathrm{G}_{2}(\mathrm{~s})=\mathrm{lG}(\mathrm{s}, \mathrm{s}),
\end{aligned}
$$


i.e.,

$$
l G(s, s) \leqslant G(t, s)
$$

Now from the definition of Green function, it is clear that

$$
G(t, s) \leqslant G(s, s) .
$$

Combining (2.9) and (2.10), we get

$$
\operatorname{lG}(s, s) \leqslant G(t, s) \leqslant G(s, s) .
$$

Remark 2.8. If we replace the Green function $G(t, s)$ by $G^{*}(t, s)$ in the Lemma 2.7, then the similar result holds, i.e., $\mathrm{lG}^{*}(\mathrm{~s}, \mathrm{~s}) \leqslant \mathrm{G}^{*}(\mathrm{t}, \mathrm{s}) \leqslant \mathrm{G}^{*}(\mathrm{~s}, \mathrm{~s})$ holds.

Lemma 2.9. Let $\mathrm{h}^{*}(\mathrm{t}) \in \mathrm{C}^{+}[0,1]$, then the unique symmetric solution $\mathrm{u}(\mathrm{t})$ of the BVP given by (2.5) is nonnegative on $[0,1]$.

Proof. Suppose $h^{*}(t) \in C^{+}[0,1]$, then from the fact $u^{(4)}(t)=-h^{*}(t) \leqslant 0, t \in[0,1]$, we confirm that the graph of $u(t)$ is concave on $[0,1]$. So, from the BVP given by (2.5), we obtain

$$
\begin{aligned}
u(0)=u(1)= & \int_{0}^{1} G(1, s)\left(\int_{0}^{1} G^{*}(1, r) h^{*}(r) d r\right) d s \\
= & \int_{0}^{1} G(1, s)\left(\frac{1}{2} \int_{0}^{1}\left[\left(t_{1}-r\right)+\left(t_{2}-r\right)-t_{1}(1-r)-t_{2}(1-r)+1\right] h^{*}(r) d r\right) d s \\
& +\int_{0}^{1} G(1, s)\left(\frac{1}{2} \int_{0}^{1}\left[\left(t_{2}-r\right)-t_{1}(1-r)-t_{2}(1-r)+1\right] h^{*}(r) d r\right) d s \\
& +\int_{0}^{1} G(1, s)\left(\frac{1}{2} \int_{0}^{1}\left[-t_{1}(1-r)-t_{2}(1-r)+1\right] h^{*}(r) d r\right) d s \geqslant 0,
\end{aligned}
$$

and since $u(t)$ is concave, thus $u(t) \geqslant 0$, for all $t \in[0,1]$. This completes the proof.

Lemma 2.10. Let $\mathrm{h}^{*}(\mathrm{t}) \in \mathrm{C}^{+}[0,1]$, then the unique symmetric solution $\mathrm{u}(\mathrm{t})$ of the BVP given by (2.5) satisfies

$$
\min _{t \in[0,1]} u(t) \geqslant l\|u\|
$$

where $\mathrm{l}$ is defined as like the Lemma 2.7.

Proof. For any $\mathrm{t} \in[0,1]$, from the Lemma 2.7 and Remark 2.8, we have

$$
G(t, s) \leqslant G(s, s) \text { and } G^{*}(t, s) \leqslant G^{*}(s, s),
$$

which implies that

$$
u(t)=\int_{0}^{1} G(t, s)\left(\int_{0}^{1} G^{*}(s, r) h^{*}(r) d r\right) d s \leqslant \int_{0}^{1} G(s, s)\left(\int_{0}^{1} G^{*}(r, r) h^{*}(r) d r\right) d s .
$$

Therefore,

$$
\|u\| \leqslant \int_{0}^{1} G(s, s)\left(\int_{0}^{1} G^{*}(r, r) h^{*}(r) d r\right) d s .
$$

Again, for any $t \in[0,1]$, from the Lemma 2.7 and Remark 2.8, we obtain

$$
l G(s, s) \leqslant G(t, s) \text { and } l^{*}(s, s) \leqslant G^{*}(t, s),
$$


which implies that

$$
u(t)=\int_{0}^{1} G(t, s)\left(\int_{0}^{1} G^{*}(s, r) h^{*}(r) d r\right) d s \geqslant l \int_{0}^{1} G(s, s)\left(\int_{0}^{1} G^{*}(r, r) h^{*}(r) d r\right) d s .
$$

Therefore,

$$
u(t) \geqslant l\|u\| .
$$

Hence, the inequalities (2.12) and (2.13), ensure that (2.11) holds.

Remark 2.11. From the Lemma 2.6, it is clear that $(u, v) \in C^{2}[0,1] \times C^{2}[0,1]$ is a solution of the SNLFOODEs given by (1.1) if and only if $(u, v) \in C[0,1] \times C[0,1]$ is the solution of the system of integral equations

$$
\left\{\begin{array}{l}
u(t)=\int_{0}^{1} G(t, s)\left(\int_{0}^{1} G^{*}(s, r) f(r, v(r)) d r\right) d s \\
v(t)=\int_{0}^{1} G^{\dagger}(t, s)\left(\int_{0}^{1} G^{\dagger *}(s, r) g(r, u(r)) d r\right) d s
\end{array}\right.
$$

If we combine the integral equations defined by (2.14), then we yield the nonlinear integral equation

$$
u(t)=\int_{0}^{1} G(t, s)\left(\int_{0}^{1} G^{*}(s, r) f\left(r, \int_{0}^{1} G^{\dagger}\left(r, s^{*}\right)\left(\int_{0}^{1} G^{\dagger *}\left(s^{*}, r^{*}\right) g\left(r^{*}, u\left(r^{*}\right)\right) d r^{*}\right) d s^{*}\right) d r\right) d s
$$

Remark 2.12. Let $K=\left\{u \in C^{+}[0,1]: u(t)\right.$ is symmetric, concave on $[0,1]$, and $\left.\min _{t \in[0,1]} u(t) \geqslant l\|u\|\right\}$. Then it is noticeable that $K$ is a positive cone in $C[0,1]$. If we define an integral operator $T: K \rightarrow C[0,1]$ by

$$
T u(t)=\int_{0}^{1} G(t, s)\left(\int_{0}^{1} G^{*}(s, r) f\left(r, \int_{0}^{1} G^{\dagger}\left(r, s^{*}\right)\left(\int_{0}^{1} G^{\dagger *}\left(s^{*}, r^{*}\right) g\left(r^{*}, u\left(r^{*}\right)\right) d r^{*}\right) d s^{*}\right) d r\right) d s,
$$

then it is clear that the BVP introduced by (1.1) and (1.2) has a symmetric positive solution $u=u(t)$ if and only if $u$ is a fixed point of the integral operator T defined by (2.15).

Lemma 2.13. The operator $\mathrm{T}: \mathrm{K} \rightarrow \mathrm{K}$ defined by (2.15) is completely continuous.

Proof. From Remark 2.12, it is clear that $T u$ is symmetric on $[0,1],(T u)^{\prime \prime}-f(t, v(t)) \leqslant 0$, and $T u$ is concave. Now applying Lemma 2.9 for $T u$, we obtain $T u \in C^{+}[0,1]$. Thus, from Lemma 2.7 and non-negativity of $f$ and $g$, we get

$$
\begin{aligned}
T u(t) & =\int_{0}^{1} G(t, s)\left(\int_{0}^{1} G^{*}(s, r) f\left(r, \int_{0}^{1} G^{\dagger}\left(r, s^{*}\right)\left(\int_{0}^{1} G^{\dagger *}\left(s^{*}, r^{*}\right) g\left(r^{*}, u\left(r^{*}\right)\right) d r^{*}\right) d s^{*}\right) d r\right) d s \\
& \leqslant \int_{0}^{1} G(s, s)\left(\int_{0}^{1} G^{*}(s, r) f\left(r, \int_{0}^{1} G^{\dagger}\left(r, s^{*}\right)\left(\int_{0}^{1} G^{\dagger *}\left(s^{*}, r^{*}\right) g\left(r^{*}, u\left(r^{*}\right)\right) d r^{*}\right) d s^{*}\right) d r\right) d s \\
& \leqslant \int_{0}^{1} G(s, s)\left(\int_{0}^{1} G^{*}(r, r) f\left(r, \int_{0}^{1} G^{\dagger}\left(r, s^{*}\right)\left(\int_{0}^{1} G^{\dagger *}\left(s^{*}, r^{*}\right) g\left(r^{*}, u\left(r^{*}\right)\right) d r^{*}\right) d s^{*}\right) d r\right) d s
\end{aligned}
$$

Hence, we obtain

$$
\|T u\| \leqslant \int_{0}^{1} G(s, s)\left(\int_{0}^{1} G^{*}(r, r) f\left(r, \int_{0}^{1} G^{\dagger}\left(r, s^{*}\right)\left(\int_{0}^{1} G^{\dagger *}\left(s^{*}, r^{*}\right) g\left(r^{*}, u\left(r^{*}\right)\right) d r^{*}\right) d s^{*}\right) d r\right) d s
$$

Again, from another part of Lemma 2.7, we get

$$
T u \geqslant l \int_{0}^{1} G(s, s)\left(\int_{0}^{1} G^{*}(r, r) f\left(r, \int_{0}^{1} G^{\dagger}\left(r, s^{*}\right)\left(\int_{0}^{1} G^{\dagger *}\left(s^{*}, r^{*}\right) g\left(r^{*}, u\left(r^{*}\right)\right) d r^{*}\right) d s^{*}\right) d r\right) d s .
$$


Hence, from (2.16) and (2.17), we have

$$
T u \geqslant l\|T u\| .
$$

Thus, $T[K] \subset K$ Now, since $G(t, s), G^{*}(t, s), G^{\dagger}(t, s), G^{\dagger *}(t, s), f(t, v(t))$ and $g(t, u(t))$ all are continuous, then it is easy to prove that the operator $T: K \rightarrow K$ is completely continuous. This completes the proof.

Now, we state two fixed point theorems of Krasnoselskii's according to Guo [10], which are crucial to our main results.

Theorem 2.14 ([10]). Let $\mathrm{B}$ be a Banach space and $\mathrm{K} \subset \mathrm{B}$ be a cone in $\mathrm{B}$. Assume that $\Psi_{1}$ and $\Psi_{2}$ are two bounded open subsets of $\mathrm{B}$ with $0 \in \Psi_{1}$ and $\bar{\Psi}_{1} \subset \Psi_{2}$. Let operator $\mathrm{T}: \mathrm{K} \cap\left(\bar{\Psi}_{2} \backslash \Psi_{1}\right) \rightarrow \mathrm{K}$ be completely continuous. Suppose that one of the following two conditions are satisfied:

$$
\|\mathrm{Tu}\| \leqslant\|\mathrm{u}\|, \forall \mathrm{u} \in \mathrm{K} \cap \partial \Psi_{1} \text { and }\|\mathrm{Tu}\| \geqslant\|\mathrm{u}\|, \forall \mathrm{u} \in \mathrm{K} \cap \partial \Psi_{2}
$$

and

$$
\|\mathrm{Tu}\| \leqslant\|\mathrm{u}\|, \forall \mathrm{u} \in \mathrm{K} \cap \partial \Psi_{2} \text { and }\|\mathrm{Tu}\| \geqslant\|\mathrm{u}\|, \forall \mathrm{u} \in \mathrm{K} \cap \partial \Psi_{1} .
$$

Then $\mathrm{T}$ has at least one fixed point $\mathrm{u}^{*} \in \mathrm{K} \cap\left(\bar{\Psi}_{2} \backslash \Psi_{1}\right)$ and $\mathrm{u}^{*}>0$.

Theorem 2.15 ([10]). Let $\mathrm{B}$ be a Banach space and $\mathrm{K} \subset \mathrm{B}$ be a cone in $\mathrm{B}$. Assume that $\Psi_{1}, \Psi_{2}$ and $\Psi_{3}$ are three bounded open subsets of $\mathrm{B}$ with $0 \in \Psi_{1}, \bar{\Psi}_{1} \subset \Psi_{2}$ and $\bar{\Psi}_{2} \subset \Psi_{3}$. Let operator $\mathrm{T}: \mathrm{K} \cap\left(\bar{\Psi}_{3} \backslash \Psi_{1}\right) \rightarrow \mathrm{K}$ be completely continuous. Suppose the following conditions are satisfied:

$$
\begin{aligned}
& \|T u\| \geqslant\|u\|, \forall u \in K \cap \partial \Psi_{1}, \\
& \|T u\| \leqslant\|u\|, T u \neq u, \forall u \in K \cap \partial \Psi_{2}, \\
& \|T u\| \geqslant\|u\|, \forall u \in K \cap \partial \Psi_{3} .
\end{aligned}
$$

Then $\mathrm{T}$ has at least two fixed points $\mathrm{u}^{*}, \mathrm{u}^{* *}$ in $\mathrm{K} \cap\left(\bar{\Psi}_{3} \backslash \Psi_{1}\right)$ and more specifically $\mathrm{u}^{*} \in \mathrm{K} \cap\left(\Psi_{2} \backslash \Psi_{1}\right), \mathrm{u}^{* *} \in$ $\mathrm{K} \cap\left(\bar{\Psi}_{3} \backslash \bar{\Psi}_{2}\right)$.

\section{Main results}

In this section, we establish three theorems which prove the existence of at least one or at least two symmetric positive solutions of the BVP given by (1.1) and (1.2).

Theorem 3.1. If following assumptions

$$
\lim _{u \rightarrow 0^{+}} \sup _{t \in[0,1]} \frac{f(t, u)}{u}=0, \quad \lim _{u \rightarrow 0^{+}} \sup _{t \in[0,1]} \frac{g(t, u)}{u}=0,
$$

and

$$
\lim _{u \rightarrow \infty} \inf _{t \in[0,1]} \frac{f(t, u)}{u}=\infty, \lim _{u \rightarrow \infty} \inf _{t \in[0,1]} \frac{g(t, u)}{u}=\infty
$$

are satisfied, then the BVP given by (1.1) and (1.2) has at least one symmetric positive solution

$$
(u, v) \in C^{2}\left([0,1], R^{+}\right) \times C^{2}\left([0,1], R^{+}\right),
$$

where $\mathrm{u}(\mathrm{t})>0, v(\mathrm{t})>0$. 
Proof. First, suppose that $\left(A_{1}\right)$ holds. Then from $\left(A_{1}\right)$, we get a number $m_{1} \in(0,1)$ such that for each $(s, u) \in[0,1] \times\left(0, m_{1}\right), f(s, u) \leqslant \lambda_{1} u, g(s, u) \leqslant \lambda_{1} u, \forall \lambda_{1}>0$ satisfies

$$
\lambda_{1} \int_{0}^{1} G(s, s) \int_{0}^{1} G^{*}(r, r) d r d s \leqslant 1, \quad \lambda_{1} \int_{0}^{1} G^{\dagger}\left(s^{*}, s^{*}\right) \int_{0}^{1} G^{\dagger *}\left(r^{*}, r^{*}\right) d r^{*} d s^{*} \leqslant 1,
$$

and for every $u \in K$ and $\|u\|=\frac{m_{1}}{2}$ from Lemma 2.7 we note that

$$
\begin{aligned}
\int_{0}^{1} G^{\dagger}\left(r, s^{*}\right) \int_{0}^{1} G^{\dagger *}\left(s^{*}, r^{*}\right) g\left(r^{*}, u\left(r^{*}\right)\right) d r^{*} d s^{*} & \leqslant \int_{0}^{1} G^{\dagger}\left(s^{*}, s^{*}\right) \int_{0}^{1} G^{\dagger *}\left(r^{*}, r^{*}\right) g\left(r^{*}, u\left(r^{*}\right)\right) d r^{*} d s^{*} \\
& \leqslant \lambda_{1} \int_{0}^{1} G^{\dagger}\left(s^{*}, s^{*}\right) \int_{0}^{1} G^{\dagger *}\left(r^{*}, r^{*}\right) u\left(r^{*}\right) d r^{*} d s^{*} \\
& \leqslant\|u\|=\frac{m_{1}}{2}<m_{1} .
\end{aligned}
$$

Hence from (2.15), we have

$$
\begin{aligned}
T u(x) & =\int_{0}^{1} G(x, s)\left(\int_{0}^{1} G^{*}(s, r) f\left(r, \int_{0}^{1} G^{\dagger}\left(r, s^{*}\right)\left(\int_{0}^{1} G^{\dagger *}\left(s^{*}, r^{*}\right) g\left(r^{*}, u\left(r^{*}\right)\right) d r^{*}\right) d s^{*}\right) d r\right) d s \\
& \leqslant \lambda_{1} \int_{0}^{1} G(s, s)\left(\int_{0}^{1} G^{*}(r, r) \int_{0}^{1} G^{\dagger}\left(r, s^{*}\right)\left(\int_{0}^{1} G^{\dagger *}\left(s^{*}, r^{*}\right) g\left(r^{*}, u\left(r^{*}\right)\right) d r^{*}\right) d s^{*} d r\right) d s \\
& \leqslant \lambda_{1}^{2} \int_{0}^{1} G(s, s) \int_{0}^{1} G^{*}(r, r) \int_{0}^{1} G^{\dagger}\left(s^{*}, s^{*}\right) \int_{0}^{1} G^{\dagger *}\left(r^{*}, r^{*}\right) u\left(r^{*}\right) d r^{*} d s^{*} d r d s \leqslant\|u\| .
\end{aligned}
$$

Now, if put $\Psi_{1}=\left\{\mathfrak{u} \in \mathrm{C}^{+}[0,1]:\|\mathrm{u}\|<\frac{\mathrm{m}_{1}}{2}\right\}$, then we have

$$
\|T u\| \leqslant\|u\|, \forall u \in K \cap \partial \Psi_{1} .
$$

Next, suppose that $\left(A_{2}\right)$ holds, then we get a number $m_{2}>\sqrt{l} m_{1}$, where $l$ is defined as Lemma 2.7, such that for each $(s, u) \in[0,1] \times\left(m_{2},+\infty\right), f(s, u) \geqslant \lambda_{2} u, g(s, u) \geqslant \lambda_{2} u, \forall \lambda_{2}>0$ satisfies

$$
\lambda_{2} l \frac{3}{2} \int_{0}^{1} G(s, s) \int_{0}^{1} G^{*}(r, r) d r d s \geqslant 1, \lambda_{2} l \frac{3}{2} \int_{0}^{1} G^{\dagger}\left(s^{*}, s^{*}\right) \int_{0}^{1} G^{\dagger *}\left(r^{*}, r^{*}\right) d r^{*} d s^{*} \geqslant 1 \text {, }
$$

and for every $u \in K$ and $\|u\|=\frac{2 m_{2}}{\sqrt{l}}$, from Lemma 2.7 and Lemma 2.10, we have

$$
\begin{aligned}
\int_{0}^{1} G^{\dagger}\left(r, s^{*}\right) \int_{0}^{1} G^{\dagger *}\left(s^{*}, r^{*}\right) g\left(r^{*}, u\left(r^{*}\right)\right) d r^{*} d s^{*} & \geqslant l^{2} \lambda_{2} \int_{0}^{1} G^{\dagger}\left(s^{*}, s^{*}\right) \int_{0}^{1} G^{\dagger *}\left(r^{*}, r^{*}\right)\|u\| d r^{*} d s^{*} \\
& =\sqrt{\imath}\|u\|\left(l \frac{3}{2} \lambda_{2} \int_{0}^{1} G^{\dagger}\left(s^{*}, s^{*}\right) \int_{0}^{1} G^{\dagger *}\left(r^{*}, r^{*}\right) d r^{*} d s^{*}\right) \\
& \geqslant \sqrt{\imath}\|u\|=2 m_{2}>m_{2} .
\end{aligned}
$$

Hence, from (2.15), we have

$$
\begin{aligned}
T u(z) & =\int_{0}^{1} G(z, s)\left(\int_{0}^{1} G^{*}(s, r) f\left(r, \int_{0}^{1} G^{\dagger}\left(r, s^{*}\right)\left(\int_{0}^{1} G^{\dagger *}\left(s^{*}, r^{*}\right) g\left(r^{*}, u\left(r^{*}\right)\right) d r^{*}\right) d s^{*}\right) d r\right) d s \\
& \geqslant l^{2} \lambda_{2}^{2} \int_{0}^{1} G(s, s) \int_{0}^{1} G^{*}(r, r) \int_{0}^{1} G^{\dagger}\left(r, s^{*}\right) \int_{0}^{1} G^{\dagger *}\left(s^{*}, r^{*}\right) u\left(r^{*}\right) d r^{*} d s^{*} d r d s \\
& \geqslant l^{3} \lambda_{2}^{2} \int_{0}^{1} G(s, s) \int_{0}^{1} G^{*}(r, r) \int_{0}^{1} G^{\dagger}\left(s^{*}, s^{*}\right) \int_{0}^{1} G^{\dagger *}\left(r^{*}, r^{*}\right)\|u\| d r^{*} d s^{*} d r d s \geqslant\|u\| .
\end{aligned}
$$


Now, if put $\Psi_{2}=\left\{u \in C^{+}[0,1]:\|u\|<\frac{2 m_{2}}{\sqrt{l}}\right\}$, then we have

$$
\|T u\| \geqslant\|u\|, \forall u \in K \cap \partial \Psi_{2} .
$$

Therefore, from (3.1), (3.2) and Theorem 2.14, we obtain that the operator $T$ has at least one positive fixed point in $\mathrm{K} \cap\left(\bar{\Psi}_{2} \backslash \Psi_{1}\right)$, i.e., the BVP given by (1.1) and (1.2) at least has one symmetric positive solution. This completes the proof.

Theorem 3.2. If following assumptions

$$
\lim _{u \rightarrow 0^{+}} \inf _{t \in[0,1]} \frac{f(t, u)}{u}=\infty, \lim _{u \rightarrow 0^{+}} \inf _{t \in[0,1]} \frac{g(t, u)}{u}=\infty,
$$

and

$$
\lim _{\mathfrak{u} \rightarrow \infty} \sup _{\mathfrak{t} \in[0,1]} \frac{f(t, u)}{\mathfrak{u}}=0, \lim _{\mathfrak{u} \rightarrow \infty} \sup _{\mathfrak{t} \in[0,1]} \frac{g(t, \mathfrak{u})}{\mathfrak{u}}=0
$$

are satisfied, then the BVP given by (1.1) and (1.2) has at least one symmetric positive solution

$$
(u, v) \in C^{2}\left([0,1], R^{+}\right) \times C^{2}\left([0,1], R^{+}\right),
$$

where $\mathrm{u}(\mathrm{t})>0, v(\mathrm{t})>0$.

Proof. First, suppose that $\left(A_{3}\right)$ holds. Then from $\left(A_{3}\right)$, we get a number $\bar{m}_{3} \in(0,1)$ such that for each $(y, u) \in[0,1] \times\left(0, \bar{m}_{3}\right), f(y, u) \leqslant \lambda_{3} u, g(y, u) \leqslant \lambda_{3} u, \forall \lambda_{3}>0$ satisfies

$$
l \frac{3}{2} \lambda_{3} \int_{0}^{1} G(s, s) \int_{0}^{1} G^{*}(r, r) d r d s \geqslant 1, \quad l \frac{3}{2} \lambda_{3} \int_{0}^{1} G^{\dagger}\left(s^{*}, s^{*}\right) \int_{0}^{1} G^{\dagger *}\left(r^{*}, r^{*}\right) d r^{*} d s^{*} \geqslant 1 .
$$

Since $g(y, 0)=0$, so from the continuity of $g(y, u)$, we obtain a number $m_{3} \in\left(0, \bar{m}_{3}\right)$ such that $g(y, u) \leqslant$ $\frac{\bar{m}_{3}}{\int_{0}^{1} G^{\dagger}(s, s) \int_{0}^{1} G^{\dagger *}(y, y) d y d s}$, for each $(y, u) \in[0,1] \times\left(0, m_{3}\right]$. Then for every $u \in K$ and $\|u\|=m_{3}$, from Lemma 2.7 we note that

$$
\begin{aligned}
& \int_{0}^{1} G^{\dagger}\left(r, s^{*}\right) \int_{0}^{1} G^{\dagger *}\left(s^{*}, r^{*}\right) g\left(r^{*}, u\left(r^{*}\right)\right) d r^{*} d s^{*} \\
& \leqslant \int_{0}^{1} G^{\dagger}\left(s^{*}, s^{*}\right) \int_{0}^{1} G^{\dagger *}\left(r^{*}, r^{*}\right) g\left(r^{*}, u\left(r^{*}\right)\right) d r^{*} d s^{*} \\
& \leqslant \int_{0}^{1} G^{\dagger}\left(s^{*}, s^{*}\right) \int_{0}^{1} G^{\dagger *}\left(r^{*}, r^{*}\right) \frac{\bar{m}_{3}}{\int_{0}^{1} G^{\dagger}\left(s^{*}, s^{*}\right) \int_{0}^{1} G^{\dagger *}\left(r^{*}, r^{*}\right) d r^{*} d s^{*}} d r^{*} d s^{*} \\
& =\bar{m}_{3} .
\end{aligned}
$$

Hence, from (2.15), we have

$$
\begin{aligned}
T u\left(x_{1}\right) & =\int_{0}^{1} G\left(x_{1}, s\right)\left(\int_{0}^{1} G^{*}(s, r) f\left(r, \int_{0}^{1} G^{\dagger}\left(r, s^{*}\right)\left(\int_{0}^{1} G^{\dagger *}\left(s^{*}, r^{*}\right) g\left(r^{*}, u\left(r^{*}\right)\right) d r^{*}\right) d s^{*}\right) d r\right) d s \\
& \geqslant l \lambda_{3} \int_{0}^{1} G(s, s)\left(\int_{0}^{1} G^{*}(r, r) \int_{0}^{1} G^{\dagger}\left(r, s^{*}\right)\left(\int_{0}^{1} G^{\dagger *}\left(s^{*}, r^{*}\right) g\left(r^{*}, u\left(r^{*}\right)\right) d r^{*}\right) d s^{*} d r\right) d s \\
& \geqslant l^{3} \lambda_{3}^{2} \int_{0}^{1} G(s, s) \int_{0}^{1} G^{*}(r, r) \int_{0}^{1} G^{\dagger}\left(s^{*}, s^{*}\right) \int_{0}^{1} G^{\dagger *}\left(r^{*}, r^{*}\right)\|u\| d r^{*} d s^{*} d r d s \geqslant\|u\| .
\end{aligned}
$$


Now, if put $\Psi_{3}=\left\{u \in C^{+}[0,1]:\|u\|<m_{3}\right\}$, then we have

$$
\|T u\| \geqslant\|u\|, \forall u \in K \cap \partial \Psi_{3} .
$$

Next, suppose that $\left(A_{4}\right)$ holds, then there exist $e_{1}>0$ and $e_{2}>0$ such that for each $\left(y_{1}, u\right) \in[0,1] \times$ $(0, \infty), f\left(y_{1}, u\right) \leqslant \lambda_{4} u+e_{1}, g\left(y_{1}, u\right) \leqslant \lambda_{4} u+e_{2}, \forall \lambda_{4}>0$ satisfies

$$
\lambda_{4} \int_{0}^{1} G(s, s) \int_{0}^{1} G^{*}(r, r) d r d s \leqslant 1, \quad \lambda_{4} \int_{0}^{1} G^{\dagger}\left(s^{*}, s^{*}\right) \int_{0}^{1} G^{\dagger *}\left(r^{*}, r^{*}\right) d r^{*} d s^{*} \leqslant 1 .
$$

Hence, for $u \in \mathrm{C}^{+}[0,1]$ from (2.15), we have

$$
\begin{aligned}
\mathrm{Tu}\left(z_{1}\right) & =\int_{0}^{1} G\left(z_{1}, s\right)\left(\int_{0}^{1} \mathrm{G}^{*}(s, r) f\left(r, \int_{0}^{1} \mathrm{G}^{\dagger}\left(r, s^{*}\right)\left(\int_{0}^{1} \mathrm{G}^{\dagger *}\left(\mathrm{~s}^{*}, \mathrm{r}^{*}\right) \mathrm{g}\left(\mathrm{r}^{*}, u\left(r^{*}\right)\right) d r^{*}\right) d s^{*}\right) d r\right) d s \\
& \leqslant \lambda_{4} \int_{0}^{1} G(s, s) \int_{0}^{1} \mathrm{G}^{*}(r, r)\left(\int_{0}^{1} \mathrm{G}^{\dagger}\left(r, s^{*}\right)\left(\int_{0}^{1} \mathrm{G}^{\dagger *}\left(s^{*}, r^{*}\right) g\left(r^{*}, u\left(r^{*}\right)\right) d r^{*}\right) d s^{*}+e_{1}\right) d r d s \\
& \leqslant \lambda_{4} \int_{0}^{1} G(s, s) \int_{0}^{1} G^{*}(r, r)\left(\int_{0}^{1} G^{\dagger}\left(r, s^{*}\right)\left(\int_{0}^{1} G^{\dagger *}\left(s^{*}, r^{*}\right) g\left(r^{*}, u\left(r^{*}\right)\right) d r^{*}\right) d s^{*}\right) d r d s+e_{3} \\
& \leqslant \lambda_{4} \int_{0}^{1} G(s, s) \int_{0}^{1} G^{*}(r, r) \int_{0}^{1} G^{\dagger}\left(s^{*}, s^{*}\right) \int_{0}^{1} G^{\dagger *}\left(r^{*}, r^{*}\right)\left(\lambda_{4} u\left(r^{*}\right)+e_{2}\right) d r^{*} d s^{*} d r d s+e_{3} \\
& \leqslant \lambda_{4}^{2} \int_{0}^{1} G(s, s) \int_{0}^{1} G^{*}(r, r) \int_{0}^{1} G^{\dagger}\left(s^{*}, s^{*}\right) \int_{0}^{1} G^{\dagger *}\left(r^{*}, r^{*}\right)\|u\| d r^{*} d s^{*} d r d s+e_{4} \\
& \leqslant\|u\|+e_{4} .
\end{aligned}
$$

Thus, $T u \leqslant\|u\|$ as $\|u\| \rightarrow \infty$.

Now, if put $\Psi_{4}=\left\{u \in B,\|u\|<m_{4}\right\}$, then for each $u \in K$ and $\|u\|=m_{4}>m_{3}$, we have

$$
\|\mathrm{Tu}\| \leqslant\|u\|, \forall \mathrm{u} \in \mathrm{K} \cap \partial \Psi_{4} .
$$

Therefore, from (3.3), (3.4) and Theorem 2.14, we obtain that the operator $T$ has at least one positive fixed point in $\mathrm{K} \cap\left(\bar{\Psi}_{4} \backslash \Psi_{3}\right)$, i.e., the BVP given by (1.1) and (1.2) at least has one symmetric positive solution. This completes the proof.

Theorem 3.3. If $\left(A_{2}\right)$ of Theorem 3.1, $\left(A_{3}\right)$ of Theorem 3.2, and following assumption

$$
\text { there exists a constant } M_{1}>0 \text {, such that } f(s, u) \leqslant \frac{M_{1}}{\int_{0}^{1} G(s, s) \int_{0}^{1} G^{*}(r, r) d r d s}
$$

for every $(s, u) \in[0,1] \times\left[\mathrm{M}_{1}, \mathrm{M}_{1}\right]$, are satisfied, then the $B V P$ given by (1.1) and (1.2) have at least two symmetric positive solutions $\left(\mathrm{u}^{*}, v^{*}\right),\left(\mathrm{u}^{* *}, v^{* *}\right) \in \mathrm{C}^{2}\left([0,1], \boldsymbol{R}^{+}\right) \times \mathrm{C}^{2}\left([0,1], \boldsymbol{R}^{+}\right)$, where $\mathrm{u}^{*}(\mathrm{t})>0, v^{*}(\mathrm{t})>0, \mathrm{u}^{* *}(\mathrm{t})>$ $0, v^{* *}(\mathrm{t})>0$.

Proof. Suppose that $\left(A_{2}\right)$ of Theorem 3.1 holds, then according to the proof of Theorem 3.1, we get

$$
\|T u\| \geqslant\|u\|, \forall u \in K \cap \partial \Psi_{5},
$$

where, $\Psi_{5}=\left\{u \in C^{+}[0,1]:\|u\|<m_{4}\right\}$ and $m_{4} \in\left(M_{2}, \infty\right), M_{2}>0$.

Now, suppose that $\left(A_{3}\right)$ of Theorem 3.2 holds, then according to the proof of Theorem 3.2, we get

$$
\|T u\| \geqslant\|u\|, \forall u \in K \cap \partial \Psi_{6},
$$

where, $\Psi_{6}=\left\{u \in C^{+}[0,1]:\|u\|<m_{5}\right\}$ and $m_{5} \in\left(0, M_{3}\right), M_{3}>0$. 
Next, suppose that $\left(A_{5}\right)$ holds and let $\Psi_{7}=\left\{u \in C^{+}[0,1]:\|u\|<M_{1}\right\}$, then for all $u \in K \cap \partial \Psi_{7}$, we have $u(s) \in\left[\mathrm{lM}_{1}, \mathrm{M}_{1}\right]$. Now, from the Lemma 2.7, Lemma 2.10 and (2.12), we get

$$
\begin{aligned}
& \int_{0}^{1} G^{\dagger}\left(s^{*}, r^{*}\right)\left(\int_{0}^{1} G^{\dagger *}\left(r^{*}, r^{*}\right) g\left(r^{*}, u\left(r^{*}\right)\right) d r^{*}\right) d s^{*} \\
& \geqslant l \int_{0}^{1} G^{\dagger}\left(s^{*}, s^{*}\right)\left(\int_{0}^{1} G^{\dagger *}\left(r^{*}, r^{*}\right) g\left(r^{*}, u\left(r^{*}\right)\right) d r^{*}\right) d s^{*} \geqslant l\|u\|,
\end{aligned}
$$

and

$$
\begin{aligned}
& \int_{0}^{1} G^{\dagger}\left(s^{*}, r^{*}\right)\left(\int_{0}^{1} G^{\dagger *}\left(r^{*}, r^{*}\right) g\left(r^{*}, u\left(r^{*}\right)\right) d r^{*}\right) d s^{*} \\
& \leqslant \int_{0}^{1} G^{\dagger}\left(s^{*}, s^{*}\right)\left(\int_{0}^{1} G^{\dagger *}\left(r^{*}, r^{*}\right) g\left(r^{*}, u\left(r^{*}\right)\right) d r^{*}\right) d s^{*} \\
& \leqslant \int_{0}^{1} G^{\dagger}\left(s^{*}, s^{*}\right) \int_{0}^{1} G^{\dagger *}\left(r^{*}, r^{*}\right) d r^{*} d s^{*} \frac{M_{1}}{\int_{0}^{1} G^{\dagger}\left(s^{*}, s^{*}\right) \int_{0}^{1} G^{\dagger *}\left(r^{*}, r^{*}\right) d r^{*} d s^{*}}=M_{1} .
\end{aligned}
$$

Hence from (2.15), we have

$$
\begin{aligned}
T u(p) & =\int_{0}^{1} G(p, s)\left(\int_{0}^{1} G^{*}(s, r) f\left(r, \int_{0}^{1} G^{\dagger}\left(r, s^{*}\right)\left(\int_{0}^{1} G^{\dagger *}\left(s^{*}, r^{*}\right) g\left(r^{*}, u\left(r^{*}\right)\right) d r^{*}\right) d s^{*}\right) d r\right) d s \\
& \leqslant \int_{0}^{1} G(s, s) \int_{0}^{1} G^{*}(r, r) f\left(r, M_{1}\right) d r d s \\
& =\int_{0}^{1} G(s, s) \int_{0}^{1} G^{*}(r, r) f(r, u) d r d s \\
& \leqslant \int_{0}^{1} G(s, s) \int_{0}^{1} G^{*}(r, r) d r d s \frac{M_{1}}{\int_{0}^{1} G(s, s) \int_{0}^{1} G^{*}(r, r) d r d s}=M_{1}
\end{aligned}
$$

i.e., $T u(p) \leqslant M_{1}$. Then for all $u \in K \cap \partial \Psi_{7}$, we have

$$
\|\mathrm{Tu}\| \leqslant\|u\| \text {. }
$$

Therefore, from (3.5), (3.6), (3.7) and Theorem 2.15, we obtain that the operator $T$ has at least two positive fixed points in $K \cap\left(\bar{\Psi}_{5} \backslash \bar{\Psi}_{7}\right)$ and $K \cap\left(\Psi_{7} \backslash \Psi_{6}\right)$, respectively, that is the BVP given by (1.1) and (1.2) at least has two symmetric positive solutions. This completes the proof.

\section{Examples}

In this section, we present some particular examples to explain our main results.

Example 4.1. Consider the following SNLFOODEs:

$$
\left\{\begin{array}{l}
-u^{(4)}(t)=v^{4}+\frac{v^{4}\left[1+t^{2}(1-t)^{2}\right]}{1+v^{4}} \\
-v^{(4)}(t)=4 u^{4}+\frac{4 u^{4}\left[1+t^{2}(1-t)^{2}\right]}{1+u^{4}}, t \in[0,1]
\end{array}\right.
$$

with the four-point boundary value conditions

$$
\left\{\begin{array}{l}
u(t)=u(1-t), u^{\prime \prime \prime}(0)-u^{\prime \prime \prime}(1)=u^{\prime \prime}\left(\frac{1}{8}\right)+u^{\prime \prime}\left(\frac{1}{4}\right), \\
v(t)=v(1-t), v^{\prime \prime \prime}(0)-v^{\prime \prime \prime}(1)=v^{\prime \prime}\left(\frac{1}{8}\right)+v^{\prime \prime}\left(\frac{1}{4}\right) .
\end{array}\right.
$$


Now, if we choose $l=\frac{5}{64}$, then all conditions of Theorem 3.1 are satisfied. Hence from Theorem 3.1, we can say that the system of BVP given by (4.1) and (4.2) has at least one symmetric positive solution.

Example 4.2. Consider the following SNLFOODEs:

$$
\left\{\begin{array}{l}
-\mathrm{u}^{(4)}(\mathrm{t})=v^{\frac{5}{2}}+\frac{v^{4}\left[1+\mathrm{t}^{2}(1-\mathrm{t})^{2}\right]}{1+v^{4}} \\
-v^{(4)}(\mathrm{t})=4 \mathrm{u}^{\frac{5}{2}}+\frac{4 \mathrm{u}^{4}\left[1+\mathrm{t}^{2}(1-\mathrm{t})^{2}\right]}{1+\mathrm{u}^{4}}, \mathrm{t} \in[0,1],
\end{array}\right.
$$

with the four-point boundary value conditions

$$
\left\{\begin{array}{l}
u(t)=u(1-t), u^{\prime \prime \prime}(0)-u^{\prime \prime \prime}(1)=u^{\prime \prime}\left(\frac{1}{8}\right)+u^{\prime \prime}\left(\frac{1}{4}\right), \\
v(t)=v(1-t), v^{\prime \prime \prime}(0)-v^{\prime \prime \prime}(1)=v^{\prime \prime}\left(\frac{1}{8}\right)+v^{\prime \prime}\left(\frac{1}{4}\right) .
\end{array}\right.
$$

Now, if we choose $l=\frac{5}{64}$, then all conditions of Theorem 3.2 are satisfied. Hence from Theorem 3.2, we can say that the system of BVP given by (4.3) and (4.4) has at least one symmetric positive solution.

Example 4.3. Consider the following SNLFOODEs:

$$
\left\{\begin{array}{l}
-\mathrm{u}^{(4)}(\mathrm{t})=\frac{85}{64}\left[1+\mathrm{t}^{2}(1-\mathrm{t})^{2}\right]\left(v^{\frac{5}{2}}+v^{4}\right), \\
-v^{(4)}(\mathrm{t})=\frac{81}{64}\left[1+\mathrm{t}^{2}(1-\mathrm{t})^{2}\right]\left(\mathrm{u}^{\frac{5}{2}}+\mathrm{u}^{4}\right), \mathrm{t} \in[0,1]
\end{array}\right.
$$

with the four-point boundary value conditions

$$
\left\{\begin{array}{l}
u(t)=u(1-t), u^{\prime \prime \prime}(0)-u^{\prime \prime \prime}(1)=u^{\prime \prime}\left(\frac{1}{8}\right)+u^{\prime \prime}\left(\frac{1}{4}\right), \\
v(t)=v(1-t), v^{\prime \prime \prime}(0)-v^{\prime \prime \prime}(1)=v^{\prime \prime}\left(\frac{1}{8}\right)+v^{\prime \prime}\left(\frac{1}{4}\right)
\end{array}\right.
$$

Now, if we choose $l=\frac{5}{64}$ and $M_{1}=1$, then all conditions of Theorem 3.3 are satisfied. Hence from Theorem 3.3, we can say that the system of BVP given by (4.5) and (4.6) has at least two symmetric positive solutions.

\section{Conclusion}

In this work, we established general approaches for checking the existence of symmetric positive solutions of SNLFOODEs for four-point boundary value conditions with the help of Krasnoselskii's fixed point theorem under suitable conditions. Theorems 3.1 and 3.2 have been used to examine the existence of single symmetric positive solution of BVP given by (1.1) and (1.2), whereas Theorem 3.3 has been used to check the existence of double symmetric positive solutions of that BVP. Corresponding three justifying examples also discussed here.

\section{Acknowledgment}

We would like to provide our sincere thank to the honourable reviewers for their valuable comments which help us to enriched the quality of this work. 


\section{References}

[1] R. P. Agarwal, On fourth-order boundary value problems arising in beam analysis, Differential Integral Equations, 2 (1989), 91-110. 1

[2] M. Asaduzzaman, M. Zulfikar Ali, Existence of three positive solutions for nonlinear third order arbitrary two-point boundary value problems, Differ. Uravn. Protsessy Upr., 2019 (2019), 83-100. 1

[3] M. Asaduzzaman, M. Zulfikar Ali, Fixed Point Theorem Based Solvability of Fourth Order Nonlinear Differential Equation with Four-point Boundary Value Conditions, Adv. Fixed Point Theory, 9 (2019), 260-272.

[4] M. Asaduzzaman, M. Zulfikar Ali, The unique symmetric positive solutions for nonlinear fourth order arbitrary two-point boundary value problems: A fixed point theory approach, Adv. Fixed Point Theory, 9 (2019), 80-98. 1

[5] E. Cetin, F. S. Topal, Symmetric positive solutions of fourth order boundary value problems for an increasing homeomorphism and homomorphism on time-scales, Comput. Math. Appl., 63 (2012), 669-678. 1

[6] S. H. Chen, W. Ni, C. P. Wang, Positive solution of fourth order ordinary differential equation with four-point boundary conditions, Appl. Math. Lett., 19 (2006), 161-168. 1

[7] E. Dulacska, Soil settlement effects on buildings, in: Developments in Geotechnical Engineering, Amsterdam, (1992). 1

[8] H. Y. Feng, D. L. Bai, M. Q. Feng, Multiple symmetric positive solutions to four-point boundary-value problems of differential systems with P-Laplacian, Electron. J. Differential Equations, 2012 (2012), 11 pages. 1

[9] M. Q. Feng, P. Li, S. J. Sun, Symmetric positive solutions for fourth-order n-dimensional m-Laplace systems, Bound. Value Probl., 2018 (2018), 20 pages. 1

[10] D. J. Guo, Nonlinear Functional Analysis, Shangdong Sci. Tech. Press, Jinan, (1985). 1, 2, 2.14, 2.15

[11] C. P. Gupta, Existence and uniqueness theorem for a bending of an elastic beam equation, Appl. Anal., 26 (1988), $289-304$. 1

[12] J. Henderson, H. B. Thompson, Multiple symmetric positive solutions for a second order boundary value problem, Proc. Amer. Math. Soc., 128 (2000), 2373-2379. 1

[13] F. Y. Li, Y. Zhang, Multiple symmetric nonnegative solutions of second-order ordinary differential equations, Appl. Math. Lett., 17 (2004), 261-267. 1

[14] Y. H. Li, X. Y. Zhang, Positive Solutions for Systems of Nonlinear Higher Order Differential Equations with Integral Boundary Conditions, Abstr. Appl. Anal., 2014 (2014), 7 pages. 1

[15] X. Z. Lv, L. B. Wang, M. H. Pei, Monotone positive solution of a fourth-order BVP with integral boundary conditions, Bound. Value Probl., 2015 (2015), 12 pages. 1

[16] P.K. Palamides, A. P. Palamides, Fourth-Order Four-Point Boundary Value Problem: A Solutions Funnel Approach, Int. J. Math. Math. Sci., 2012 (2012), 18 pages. 1

[17] H. D. Qu, The Symmetric Positive Solutions of Four-Point Problems for Nonlinear Boundary Value Second-Order Differential Equations, Int. J. Math. Anal. (Ruse), 3 (2009), 1969-1979. 1

[18] H. D. Qu, The symmetric positive solutions of three-point boundary value problems for nonlinear second-order differential equations, Bull. Inst. Math. Acad. Sin. (N.S.), 7 (2012), 405-416. 1, 2.1, 2.3, 2.4

[19] W. Soedel, Vibrations of Shells and Plates, Dekker, New York, (1993). 1

[20] Y. P. Sun, Existence and multiplicity of symmetric positive solutions for three-point boundary value problem, J. Math. Anal. Appl., 329 (2007), 998-1009. 1

[21] S. P. Timoshenko, Theory of Elastic Stability, McGraw-Hill, New York, (1961). 1

[22] M. Tuz, The Existence of Symmetric Positive Solutions of Fourth-Order Elastic Beam Equations, Symmetry, 11 (2019), 14 pages. 1

[23] A. K. Verma, N. Urus, Region of existence of multiple solutions for a class of 4-point BVPs, arXiv, 2019 (2019), 21 pages. 1

[24] J. R. L. Webb, G. Infante, D. Franco, Positive solutions of nonlinear fourth-order boundary-value problems with local and non-local boundary conditions, Proc. Roy. Soc. Edinburgh Sect. A, 138 (2008), 427-446. 1

[25] Q. L. Yao, Existence and iteration of $n$ symmetric positive solutions for a singular two-point boundary value problem, Comp. Math. Appl., 47 (2004), 1195-1200. 1

[26] C. Zhai, R. Song, Q. Han, The existence and the uniqueness of symmetric positive solutions for a fourth-order boundary value problem, Comput. Math. Appl., 62 (2011), 2639-2647. 2.1

[27] X. Y. Zhang, Fixed point theorems for a class of nonlinear operators in Banach spaces and applications, Nonlinear Anal., 69 (2008), 536-543.

[28] X. M. Zhang, M. Q. Feng, W. G. Ge, Symmetric positive solutions for p-Laplacian fourth-order differential equations with integral boundary conditions, J. Comput. Appl. Math., 222 (2008), 561-573. 1

[29] D. G. Zill, M. R. Cullen, Differential Equations with Boundary-Value Problems, Brooks/Cole, Boston, (2001). 1 